\title{
Optimal surveillance and treatment of renal and splenic artery aneurysms
}

\section{ABSTRACT}

Aneurysms of the renal artery and splenic artery are uncommon but clinically important, as they pose a risk of rupture with a high fatality rate. Indications for surgical or endovascular repair are based on aneurysm location and risk factors for rupture, such as aneurysm size, growth, and associated conditions, while medical management is also important. Regular surveillance with imaging is critical before and after intervention to guide treatment.

\section{KEY POINTS}

Renal and splenic artery aneurysms are often detected incidentally but can present acutely with dissection, rupture, or both, which are associated with high risk of death and morbidities.

Computed tomographic and magnetic resonance angiography are key to diagnosing and characterizing the aneurysm and the remaining vasculature, while ultrasonography helps in assessment and surveillance. Catheter angiography is the gold standard for diagnosis and allows the opportunity for intervention.

The individual's risk for rupture or dissection determines the need for prophylactic intervention and is based on aneurysm size, location, growth, and other associated conditions and risk factors.

Management strategies include open and laparoscopic surgery and endovascular procedures. Regular imaging surveillance is critical after both diagnosis and interventions.

The authors report no relevant financial relationships which, in the context of their contributions, could be perceived as a potential conflict of interest.

doi:10.3949/ccjm.87a.19140-2
Lthough aneurysms of the abdominal $A$ and thoracic aorta are more common, visceral aneurysms such as those of the renal artery and splenic artery can also form.

In a previous article, ${ }^{1}$ we discussed the optimal surveillance strategies and treatment for aneurysm of the thoracic aorta. Here, we address the diagnosis, surveillance, and treatment of renal artery and splenic artery aneurysms.

\section{RENAL ARTERY ANEURYSM}

What is the clinical importance of renal artery aneurysm?

Renal artery aneurysms are rare, found in about $0.1 \%$ of the population in autopsy studies, although significantly more in cross-sectional imaging or angiography studies. ${ }^{2}$ They are categorized by location and morphology into 3 types (Figure 1), with important management implications:

- Type 1-saccular aneurysm from the main renal artery or large segmental branch

- Type 2-fusiform aneurysm

- Type 3-intralobar artery aneurysm. ${ }^{3}$

Renal artery aneurysms often present in the sixth decade. Up to $90 \%$ of patients have hypertension, and a minority have arterial aneurysms elsewhere. ${ }^{4}$ Most younger patients with renal artery aneurysms are women, and about two-thirds have fibromuscular dysplasia.

Symptoms are rare but can include hypertensive crisis, shock, hematuria, flank and abdominal pain, and urinary obstruction, with signs of a palpable abdominal mass and a renal bruit. ${ }^{2}$ The natural history is slow growth at 0.06 to $0.09 \mathrm{~mm}$ per year. The clinical concern is rupture, which occurs in 3\% to $5 \%$, with mortality rates near $10 \%$, although these figures are lower than with other types of visceral aneurysms. ${ }^{2,5}$ 


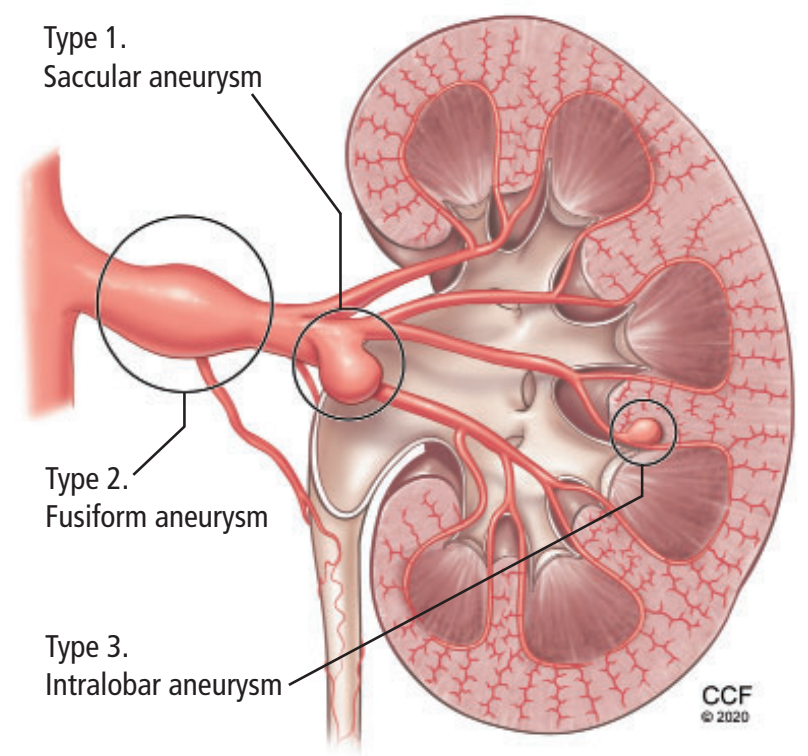

Figure 1. Three types of renal artery aneurysms.

How is renal artery aneurysm assessed?

Renal artery aneurysms are often diagnosed incidentally either during investigation of resistant hypertension or during angiography for another indication. They are detected more often now than in the past because of improvements in imaging. ${ }^{2}$

Blood pressure and renal function should be routinely assessed. Accurate measurement is important, as size is directly associated with risk of rupture and need for intervention. Circumferential calcification may be protective. ${ }^{2}$

Duplex ultrasonography is the least expensive imaging modality. Further, it does not expose the patient to radiation, and it can show a dilated vessel with turbulent flow. On the other hand, it has the lowest sensitivity and specificity, and its accuracy depends on operator experience and the patient's body habitus. ${ }^{6}$

Multidetector computed tomographic angiography (CTA) is the most commonly used modality for assessing renal artery aneurysms. Its superior resolution can characterize anatomy using multiplanar reconstructions and volume-rendered imaging (Figure 2). On the negative side, it entails the use of nephrotoxic contrast media and radiation exposure. ${ }^{7}$

Magnetic resonance angiography (MRA) has become an alternative to CTA, with comparable accuracy and without radiation, but it is less available and costs more. ${ }^{6,7}$
Catheter angiography remains the gold standard invasive option for diagnosing renal artery aneurysm, allowing assessment of proximal and distal aneurysms, and providing the opportunity for percutaneous interventions. ${ }^{2}$

If a renal artery aneurysm is found but does not meet the criteria for intervention (see below), repeat imaging at 1, 6, and 12 months and then annually has been recommended by some experts, although guidelines are lacking. ${ }^{2}$ Ultrasonography can be used for surveillance, and abnormalities can be confirmed by any of the other 3 modalities. Annual surveillance should also be considered after surgery at least initially, and long-term after endovascular therapy. ${ }^{2,8}$

\section{How should renal artery aneurysm be treated?}

There are no guidelines for treatment of renal artery aneurysm, and if the aneurysm has not ruptured, indications for prophylactic surgery are based on rupture risk. ${ }^{2}$ These include any of the following ${ }^{4}$ :

- Large size $(>2 \mathrm{~cm})$

- Symptoms

- Refractory hypertension with significant renal artery stenosis or thromboembolism

- Childbearing age, for women.

The two main intervention options are surgical and endovascular repair. Acute rupture calls for emergency surgical repair of the aneurysm with renal artery reconstruction or with nephrectomy if the kidneys are not salvageable. For elective management, surgical primary repair or endovascular repair with stents or coil occlusions can be performed for type 1 aneurysm; surgical reconstruction with a vein graft or aortorenal bypass graft typically for type 2 aneurysm; and embolization coils for type 3 aneurysm, with renal preservation whenever possible. ${ }^{2,9}$

Mortality rates during elective interventions have been less than 5\% in recent studies, but up to $50 \%$ in pregnancy. Surgical complications include occlusion of the renal artery, branch, or graft; renal ischemia; and cardiac events. Endovascular complications include failed procedure $(<10 \%)$, thrombosis, embolization, and postembolization syndrome. ${ }^{2,10}$ "Postembolization syndrome" refers to a constellation of symptoms including abdominal pain, fever, and at times ileus and pancreatitis that occur in up to $30 \%$ of patients after ab- 


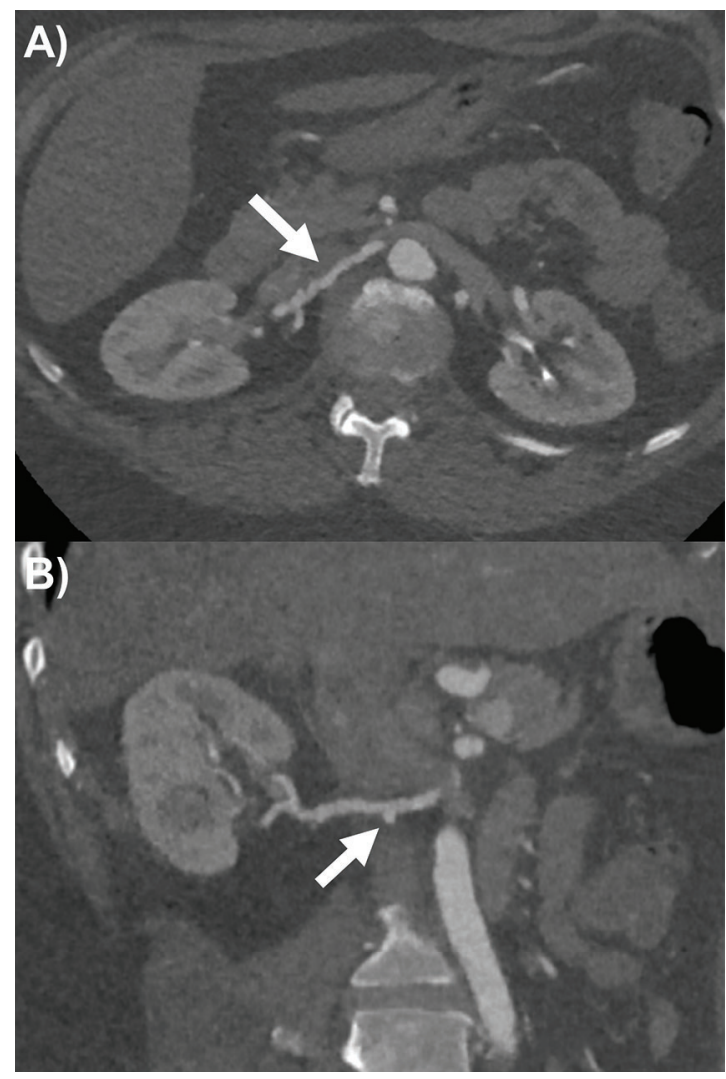

Figure 2. Right renal artery ectasia and beading with focal aneurysm (arrows) by computed tomography angiography in patient with fibromuscular dysplasia on (A) axial slice and (B) double oblique multiplanar reconstruction.

dominal visceral arterial embolization. ${ }^{11}$

Overall, outcomes have improved significantly over time with earlier detection and better planning and interventional techniques. ${ }^{2,4}$

\section{SPLENIC ARTERY ANEURYSM}

\section{What is the clinical importance} of splenic artery aneurysm?

The prevalence of splenic artery aneurysm is $0.04 \%$ to $0.10 \%$ at arteriography and autopsy. It is often found incidentally, but accounts for about $60 \%$ of visceral arterial aneurysms. ${ }^{12}$

The main risk factors or causes include portal hypertension, liver transplant, pregnancy, pancreatitis, atherosclerosis, hypertension, connective tissue disease (eg, Marfan syndrome), vasculitis, endocarditis, fibromuscular dysplasia, trauma, congenital anomalies, infection, older age, and female sex (especially

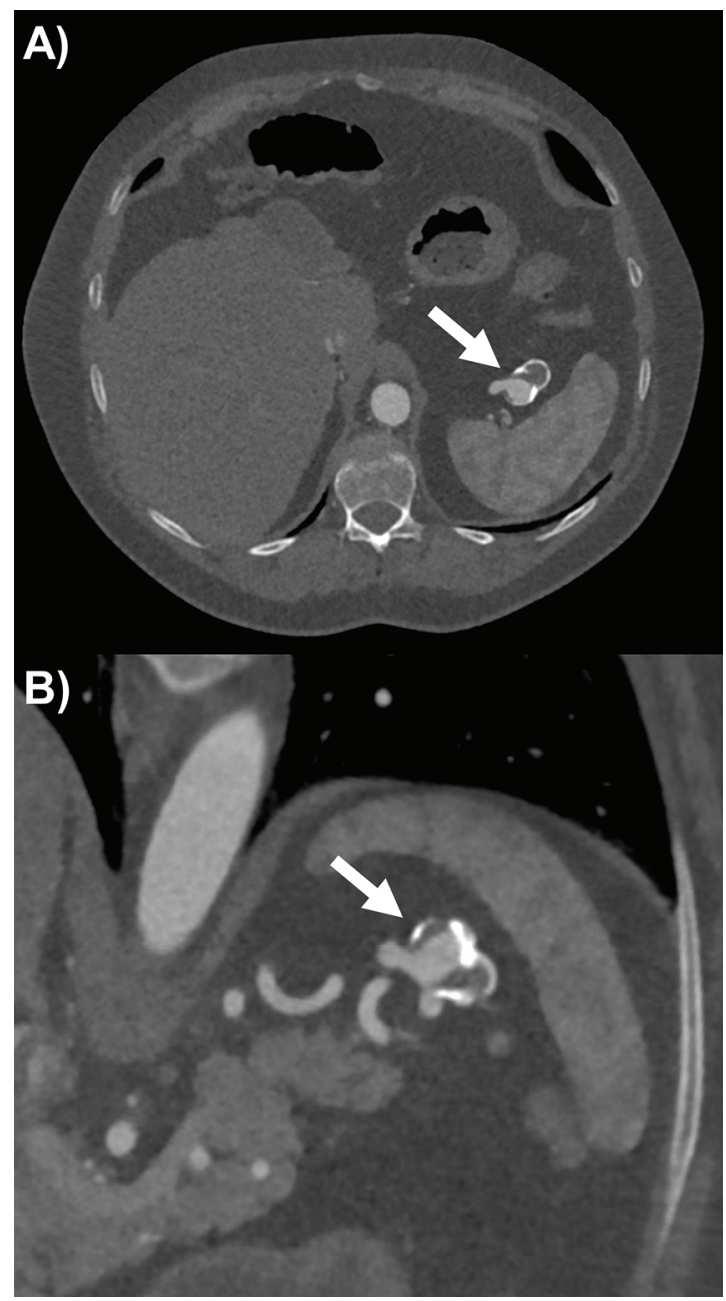

Figure 3. Splenic artery aneurysm (arrows) partially calcified and thrombosed by computed tomography angiography on (A) axial slice and (B) double oblique multiplanar reconstruction showing proximal and distal end of vessel.

multiparous women). ${ }^{11,12}$

Symptoms occur in up to $20 \%$ of cases and include upper abdominal pain that can radiate to the shoulder, nausea, vomiting, anorexia, and gastrointestinal bleeding. Rupture presents with acute abdomen, peritoneal bleeding, and shock. ${ }^{12,13}$ The risk of rupture is $2 \%$ to $10 \%$, with a mortality rate of $25 \%$, and both figures are markedly higher in pregnancy. ${ }^{12,14}$

\section{How should splenic artery aneurysm be evaluated?}

Abdominal radiographs rarely detect splenic artery aneurysm. Ultrasonography has some utility as an accessible, low-cost, and radi-

\section{Outcomes}

have improved with earlier detection and better planning and interventional techniques 
ation-free tool, with varying sensitivity for splenic artery aneurysm depending on the operator's experience and the patient's body habitus. ${ }^{12}$ Therefore, CTA (Figure 3) and MRA are again the preferred imaging modalities for diagnosing splenic artery aneurysms. Both can provide 3-dimensional reconstruction for accurately assessing the aneurysm's dimensions, the neighboring vasculature, and other abdominal pathologies that could contribute to its cause. ${ }^{12,13}$ Endoscopic ultrasonography can also assist in the diagnosis and differentiate splenic artery aneurysm from nearby splenic and pancreatic pathology, such as pancreatic pseudocyst. ${ }^{13,15}$ Catheter angiography, although invasive, continues to be the gold standard for characterizing the aneurysm's location, size, and extent. ${ }^{12,13}$

If intervention is not planned, surveillance is recommended at 6 months after diagnosis and then annually. Ultrasonography is suitable for surveillance if it can adequately characterize the aneurysm. CTA is also an option. ${ }^{12}$

\section{How should splenic artery aneurysm be managed?}

In the absence of guidelines, the main recommended indications for intervention of splenic artery aneurysm are rupture, aneurysm size larger than 2 or $2.5 \mathrm{~cm}$, growth of the aneu- rysm by 3 to $5 \mathrm{~mm}$ or more during surveillance regardless of initial size, symptoms, women of childbearing age, portal hypertension, and planned liver transplant. ${ }^{11,12}$

The treatment options again are open surgery (mandatory in the setting of rupture), endovascular procedures, and laparoscopic surgery.

Open surgical procedures include ligation of the splenic artery or aneurysm and aneurysmectomy with or without splenectomy. Mortality rates are around $1 \%$.

Endovascular options include transcatheter embolization, covered stent-graft insertion, and coil or thrombin injection or both, and these have now become first-line for elective management when anatomically feasible because of lower mortality and complication rates than with open surgery. ${ }^{16}$

Minimally invasive laparoscopic surgery has become an alternative. It is associated with less pain and shorter length of stay compared with open surgery, and it allows splenectomy and distal pancreatectomy to be performed if needed. ${ }^{13}$

Potential complications of intervention include postembolization syndrome, splenic infarction or abscess, and pancreatitis. Therefore, follow-up imaging with CTA or ultrasonography is recommended. ${ }^{12}$

\section{REFERENCES}

1. Wang TKM, Desai MY. Thoracic aortic aneurysm: optimal surveillance and treatment. Cleve Clin J Med 2020; 87(9):557-568. doi:10.3949/ccjm.87a.19140-1

2. González J, Esteban M, Andrés G, Linares E, Martínez-Salamanca Jl. Renal artery aneurysms. Curr Urol Rep 2014; 15(1):376. doi:10.1007/s11934-013-0376-z

3. Rundback JH, Rizvi A, Rozenblit GN, et al. Percutaneous stent-graft management of renal artery aneurysms. J Vasc Interv Radiol 2000; 11(9):1189-1193. doi:10.1016/s1051-0443(07)61362-1

4. Coleman DM, Stanley JC. Renal artery aneurysms. J Vasc Surg 2015; 62(3):779-785. doi:10.1016/j.jvs.2015.05.034

5. Wayne EJ, Edwards MS, Stafford JM, Hansen KJ, Corriere MA. Anatomic characteristics and natural history of renal artery aneurysms during longitudinal imaging surveillance. J Vasc Surg 2014; 60(2):448-452. doi:10.1016/j.jvs.2014.03.006

6. Chadha $\mathbf{M}$, Ahuja $\mathbf{C}$. Visceral artery aneurysms: diagnosis and percutaneous management. Semin Intervent Radiol 2009; 26(3):196-206. doi:10.1055/s-0029-1225670

7. Chimpiri AR, Natarajan B. Renal vascular lesions: diagnosis and endovascular management. Semin Intervent Radiol 2009; 26(3):253-261. doi:10.1055/s-0029-1225665

8. Gandini R, Morosetti D, Chiocchi M, et al. Long-term follow-up of endovascular treatment of renal artery aneurysms with covered stent deployment. J Cardiovasc Surg (Torino) 2016:57(5):625-633. pmid:25475915

9. English WP, Pearce JD, Craven TE, et al. Surgical management of renal artery aneurysms. J Vasc Surg 2004; 40(1):53-60. doi:10.1016/j.jvs.2004.03.024
10. Cochennec F, Riga CV, Allaire E, et al. Contemporary management of splanchnic and renal artery aneurysms: results of endovascular compared with open surgery from two European vascular centers. Eur J Vasc Endovasc Surg 2011; 42(3):340-346. doi:10.1016/j.ejvs.2011.04.033

11. Al-Habbal Y, Christophi C, Muralidharan V. Aneurysms of the splenic artery-a review. Surgeon 2010; 8(4):223-231. doi:10.1016/j.surge.2009.11.011

12. Madoff DC, Denys A, Wallace MJ, et al. Splenic arterial interventions: anatomy, indications, technical considerations, and potential complications. Radiographics 2005; 25(suppl 1):S191-S211. doi:10.1148/rg.25si055504

13. Kassem MM, Gonzalez L. Splenic artery aneurysm. In: StatPearls. Treasure Island, FL: StatPearls Publishing; 2020.

14. Mattar SG, Lumsden AB. The management of splenic artery aneurysms: experience with 23 cases. Am J Surg 1995; 169(6):580-584. doi:10.1016/s0002-9610(99)80225-6

15. Fang G, Chen B, Fu W, et al. Strategies for endovascular treatment of complicated splenic artery aneurysms. J Vasc Surg 2018; 68(3):787-794. doi:10.1016/j.jvs.2017.12.053

16. Hogendoorn W, Lavida A, Hunink MG, et al. Open repair, endovascular repair, and conservative management of true splenic artery aneurysms. J Vasc Surg 2014; 60(6):1667-1676.e1. doi:10.1016/j.jvs.2014.08.067

Address: Tom Kai Ming Wang, MD, Section of Cardiovascular Imaging, J15, Cleveland Clinic, 9500 Euclid Avenue, Cleveland, OH 44195; wangt2@ccf.org 